\title{
CD200 is induced by ERK and is a potential therapeutic target in melanoma
}

\author{
Kimberly B. Petermann, ${ }^{1}$ Gabriela I. Rozenberg, ${ }^{1}$ Daniel Zedek, ${ }^{2}$ Pamela Groben, ${ }^{2,3}$ \\ Karen McKinnon, ${ }^{4}$ Christin Buehler, ${ }^{4}$ William Y. Kim, ${ }^{1,5}$ Janiel M. Shields, ${ }^{6}$ Shannon Penland, ${ }^{5}$ \\ James E. Bear, ${ }^{7}$ Nancy E. Thomas, ${ }^{3}$ Jonathan S. Serody, ${ }^{4,5}$ and Norman E. Sharpless ${ }^{1,5}$
}

${ }^{1}$ Department of Genetics, ${ }^{2}$ Department of Pathology and Laboratory Medicine, ${ }^{3}$ Department of Dermatology, ${ }^{4}$ Department of Microbiology and Immunology, ${ }^{5}$ Department of Medicine, ${ }^{6}$ Department of Pharmacology, and 7 Department of Cell and Developmental Biology,

The Lineberger Comprehensive Cancer Center, University of North Carolina School of Medicine, Chapel Hill, North Carolina, USA.

\begin{abstract}
Immune-mediated antitumor responses occur in patients with metastatic melanoma (MM), and therapies designed to augment such responses are clinically beneficial. Despite the immunogenicity of melanoma, immunomodulatory therapies fail in the majority of patients with MM. An inability of DCs to sufficiently activate effector cells may, in part, underlie this failure of the antitumor response seen in most patients. In this work, we show that mutation of N-RAS or B-RAF, signature genetic lesions present in most MMs, potently induced the expression of cell-surface CD200, a repressor of DC function. Employing 2 independent, genomewide microarray analyses, we identified $C D 200$ as a highly dynamic, downstream target of RAS/RAF/MEK/ ERK activation in melanoma. CD200 protein was similarly overexpressed in human melanoma cell lines and primary tumors. CD200 mRNA expression correlated with progression and was higher in melanoma than in other solid tumors or acute leukemia. Melanoma cell lines expressing endogenous CD200 repressed primary T cell activation by DCs, while knockdown of CD200 by shRNA abrogated this immunosuppressive effect. These data indicate that in addition to its effects on growth, survival, and motility, ERK activation in MM attenuates a host antitumor immune response, implicating CD200 and its interaction with the CD200 receptor as a potential therapeutic target for MM.
\end{abstract}

\section{Introduction}

Melanoma, the most lethal form of skin cancer, has increased in incidence and mortality over the last 3 decades. Metastatic disease that is not amenable to surgery is generally refractory to therapy and, therefore, ultimately lethal. Standard chemotherapy typically produces response rates on the order of $10 \%$, and radiotherapy plays only a limited role in disease palliation. Despite these sobering facts, some optimism has been engendered by recent advances in our molecular understanding of the disease, particularly the finding that approximately $80 \%$ of metastatic melanomas (MMs) harbor mutually exclusive activating mutations of either N-RAS or B-RAF (reviewed in ref. 1). These lesions lead to activation of the RAF/MEK/ERK/MAPK pathway, which in turn controls the transcription of hundreds if not thousands of genes related to cellular proliferation, survival, and motility (2). Although work in murine models and pharmacological approaches have suggested that RAS-RAF activation is required not only for tumor formation, but also for tumor maintenance $(3,4)$, the cell-biological effects of ERK activation that are most relevant for tumor formation and progression have not been fully established.

Arguably, the evidence for a clinically valuable anticancer immune response is stronger in MM than any other human malignancy (reviewed in refs. 5-8). Functional T cells restricted to melanoma antigens can be readily recovered from patients with $\mathrm{MM}$, establishing the tumor's immunogenicity in humans $(5-7,9)$.

Nonstandard abbreviations used: CD200R, CD200 receptor; FFPE, formalin-fixed, paraffin-embedded; IHC, immunohistochemistry; MLR, mixed lymphocyte reaction; MM, metastatic melanoma; shRNA, short hairpin RNA.

Conflict of interest: The authors have declared that no conflict of interest exists. Citation for this article: J. Clin. Invest. 117:3922-3929 (2007). doi:10.1172/JCI32163.
Anecdotal spontaneous remissions, thought to be immune mediated, have been described by multiple investigators, and the appearance of vitiligo, an autoimmune response to melanocytes, is of good prognostic significance in patients with MM (10-12). Therapeutic strategies to augment the immune response, e.g., treatment with interferon and IL-2, demonstrate efficacy in certain clinical settings $(5,6,8,13)$, and anti-CTLA4 antibodies, which enhance $\mathrm{T}$ cell activation, have been reported to possess promising single-agent activity in early clinical trials $(14,15)$. Despite these findings, however, the majority of patients with MM eventually fail immunotherapeutic approaches and succumb to progressive disease. In particular, antigen-presenting cells, especially DCs, appear unable to sufficiently augment the antimelanoma response for effective tumor clearance. Melanoma cell lines have been reported to repress DC function through the elaboration of soluble factors and by direct physical interaction (16-19). These observations have in turn motivated clinical strategies to augment DC function in melanoma in order to enhance antitumor immunity $(20,21)$.

Given this background, we considered our recent finding that CD200 mRNA correlates with ERK activation in melanoma particularly provocative (2). CD200, initially described as the Ox-2 tumor antigen, is a type I membrane-associated glycoprotein and a member of the immunoglobulin superfamily (22). It is expressed on a variety of cell types, including myeloid cells, endothelium, ovarian cells, placental trophoblasts, and neurons. Recent work has shown that CD200 induces an inhibitory signal by interacting with CD200 receptors (CD200Rs) expressed on myeloid cells, particularly macrophages and DCs (23-25). This interaction generates signals that negatively regulate immune and inflammatory responses and prevent an autoimmune response in a number of systems (25-29). Moreover, a few phylogenetically distinct 
A

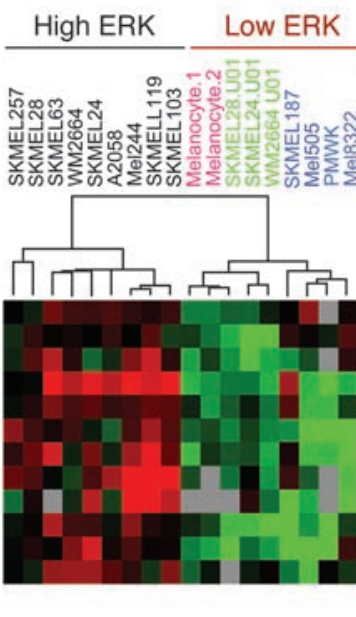

C
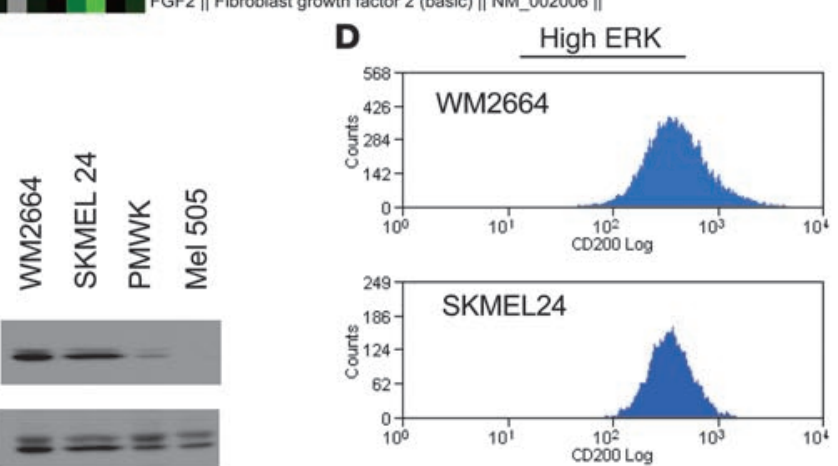

Total ERK

$\beta$-actin

CDK6 || Cyclin-dependent kinase 6 || NM_001259 || CDC6 || CDC6 cell division cycle 6 homolog || NM_001254 || NSE2 || Breast cancer membrane protein 101 || NM_174911 || COTL1 || Coactosin-like 1 (Dictyostelium) || NM_021149 || ICAM1 || Intercellular adhesion molecule 1 (CD54)|| NM_000201 MT1X || Metallothionein 1X || NM 005952 || MTIX $\|$ Metallothior CXCL1 || Chemokine (C-X-C motif) ligand 1 L_8 || Interleukin 8 || NM_000584 || SPRY2 || Sprouty homolog 2 (Drosophila) || NM_005842 || CD200 || CD200 antigen || NM 001004196 GF2 || Fibroblast growth factor 2 (basic) || NM_002006 ||

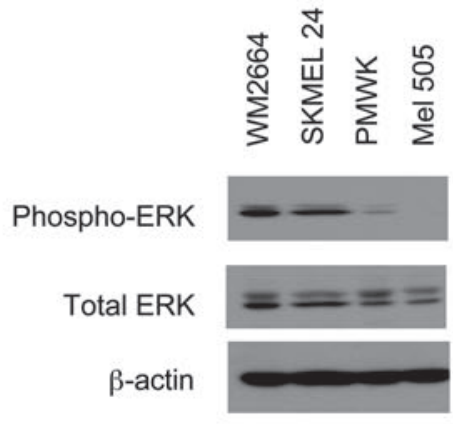
RIS1 || Ras-induced senescence 1 || NM_015444 ||

B
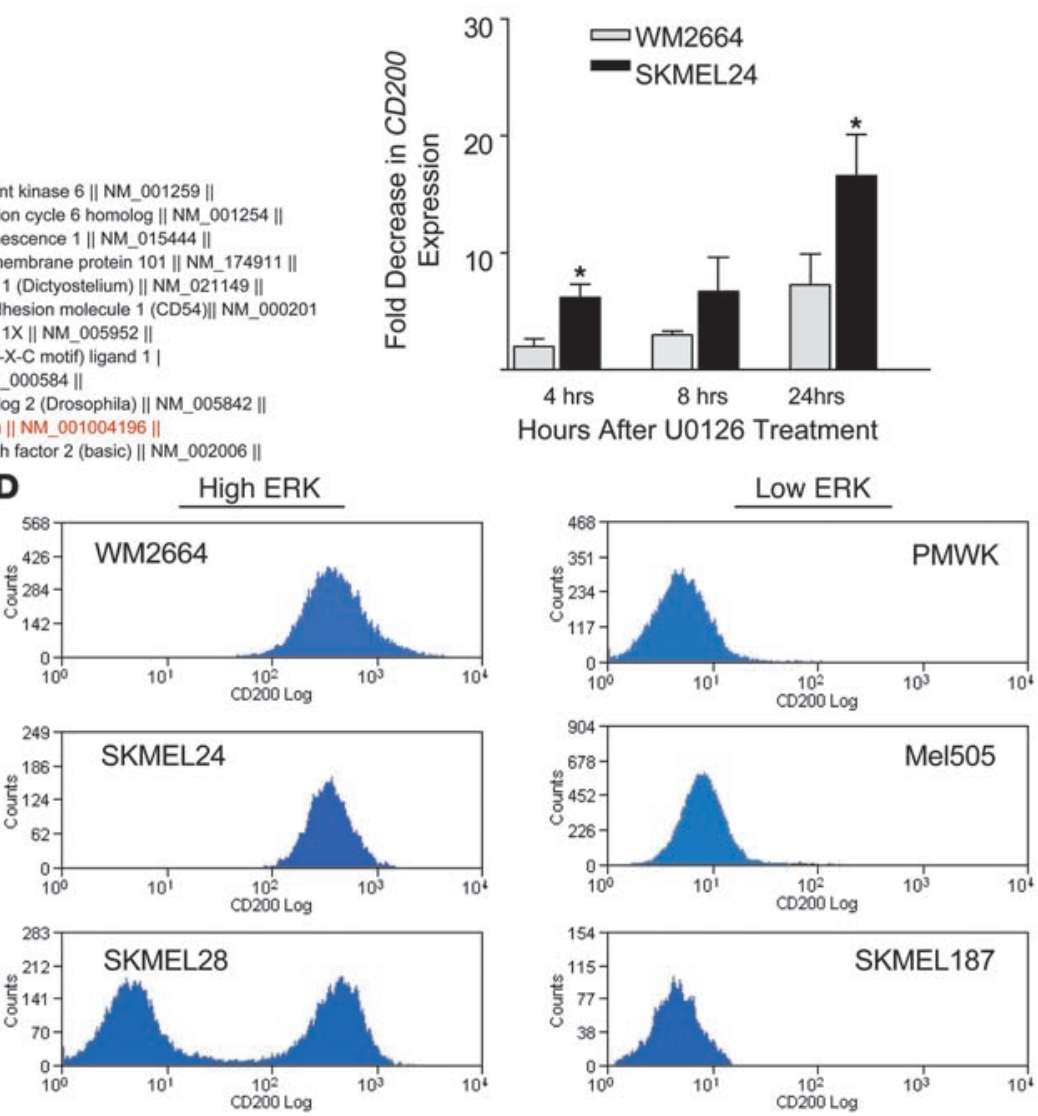

\section{Figure 1}

CD200 is a p-ERK target in melanoma. (A) Dendrogram of melanoma-specific transcripts that are regulated by p-ERK expression in indicated melanoma cell lines. Melanoma cell lines with high levels of activated ERK (black) show increased CD200 expression. Melanoma cell lines with low ERK activity lacking RAS/RAF mutation (blue) or treated with U0126 (a MEK inhibitor; green) and normal human melanocytes (red) have low expression of CD200 and other known ERK targets. (B) TaqMan qRT-PCR of CD200 expression after U0126 treatment of human melanoma cell lines. WM2664 and SKMEL24 cell lines were treated with U0126 for 4, 8, and 24 hours. CD200 expression was normalized to a control transcript (18S). Error bars are \pm SEM. ${ }^{\star} P<0.05$. (C) Immunoblot showing ERK activation in melanoma cell lines. Protein lysates from the indicated melanoma cell lines were immunoblotted with anti-p-ERK and anti-total ERK. (D) Flow cytometry was performed on melanoma cell lines WM2664, SKMEL24, and SKMEL28 (high p-ERK) and melanoma cell lines PMWK, Mel505, and SKMEL187 (low p-ERK). The high p-ERK lines express high levels of CD200, while low p-ERK lines express low levels of CD200.

viruses including poxviruses and KSHV, the causative agent of Kaposi sarcoma and other human cancers, have been shown to attenuate the host antiviral immune response by expressing a viral CD200 homolog (30-33). Given the storied history of hostgene homologs in the ability of DNA tumor viruses to promote cancer (e.g., refs. 34-36), we considered this latter finding compelling. Therefore, although 81 other ERK targets, including several of likely pathogenic significance (e.g., IL-8, TWIST1, FGF2, CXCL1/GRO1), were identified in our genomic screen, these facts motivated more detailed study of CD200 in particular. In this work, we show that CD200 mRNA and protein are regulated by ERK activation, and CD200 is expressed in the majority of melanoma but only rarely in other solid tumors. Moreover, this expression is immunologically significant, as endogenous levels of CD200 on melanoma cell lines attenuate the ability of DCs to activate $\mathrm{T}$ cells in a mixed lymphocyte reaction (MLR). As block- ing antibodies to CD200 are in preclinical development, these observations suggest that the CD200-CD200R interaction is a therapeutic target in melanoma.

\section{Results}

CD200 is regulated by the N-RAS/B-RAF/MEK/ERK/MAPK pathway. Through the manipulation of ERK signaling in melanoma cell lines, we recently demonstrated in 2 independent microarray screens that CD200 mRNA expression in melanoma is regulated by the N-RAS/B-RAF/MEK/ERK pathway (2). The pattern of CD200 mRNA expression in melanoma cell lines was similar to that of other known ERK targets such as IL-8 and SPRY2: low in normal melanocytes and melanoma cells lines with low p-ERK; high in cell lines with high expression of p-ERK; and inhibited by U0126, a potent MEK inhibitor (Figure 1A). By TaqMan RT-PCR, we showed a significant reduction in CD200 mRNA in 2 melanoma 
A

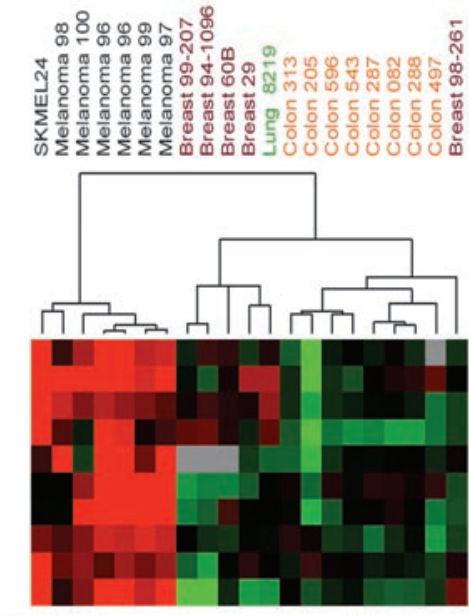

B

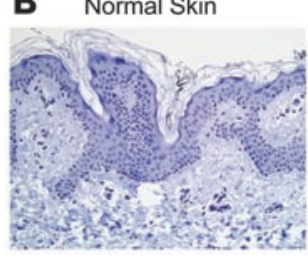

Nevus

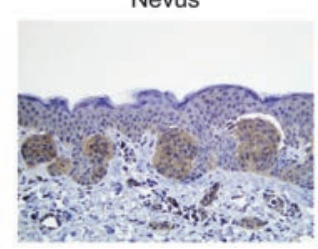

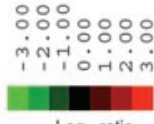

$\log _{2}$ ratio
EDNRB || Endothelin receptor type B || AF114165 S100B || S100 calcium binding protein, beta (neural) || BC001766 CD200 || CD200 antigen || NM_005944

MCAM || Melanoma cell adhesion molecule || AF0898668

DCT || Dopachrome tautomerase (tyrosine-related || A.J132932 SILV || Silver homolog (mouse) || BC001414

MITF || Microphthalmia-associated transcription factor || AL110195 PIR || Pirin (iron-binding nuclear protein) || NM_003662 COTL1 || Coactosin-like 1 (Dictyostelium) || BC010039

PRAME || Preferentially expressed antigen in melanoma || NM_006115

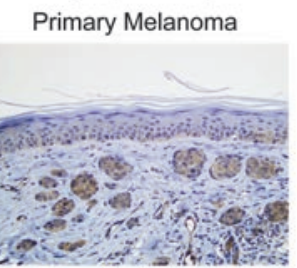

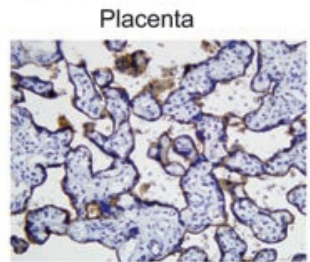

Figure 2

CD200 is expressed on melanoma. (A) Dendogram of select melanoma-associated transcripts. CD200 expression correlates with other well-established melanoma transcripts (MITF, DCT, SILV, EDNRB). $C D 200$ expression is low or absent in colon and most breast cancers. SKMEL24 human melanoma cell line was used as control. (B) IHC for CD200 was performed on a series of normal skin, nevi, and melanomas (primary and metastatic). Normal skin and melanocytes do not show CD200 expression, while the majority of nevi and melanomas strongly express CD200. Placenta is shown as a positive control; original magnification, $\times 200$. cell lines with high baseline p-ERK (WM2664 and SKMEL24) after as little as 4 hours of treatment with UO126, a pharmacologic inhibitor of MEK (Figure 1B). In addition, melanoma cell lines WM2664, SKMEL24, and SKMEL28 transduced with a mutant BRAF knockdown showed a significant reduction in CD200 mRNA expression 4 days after transduction (Supplemental Figure 1A; supplemental material available online with this article; doi:10.1172/ JCI32163DS1). Analysis of a panel of melanoma cell lines with high levels of p-ERK showed a greater than 10-fold increase in CD200 protein expression by flow cytometry when compared with melanoma cells with low or absent p-ERK (Figure 1, C and D). These data show that CD200 mRNA and protein are dynamic and highly expressed ERK targets in melanoma cell lines.

CD200 is bighly expressed on melanomas. To support these observations, the expression of CD200 mRNA and protein was tested in groups of resected human tumors. Using a newly developed method to perform expression analysis on RNA derived from formalin-fixed, paraffin-embedded (FFPE) samples (37), we extracted RNA of sufficient quality for microarray analysis from 20 solid tumors, including 6 melanoma metastases, 5 breast cancers, 8 colon cancers, and 1 lung adenocarcinoma. Through this approach, CD200 mRNA was found to be highly expressed in melanomas compared with other solid tumors, which have low or absent expression of CD200 mRNA (Figure 2A). Furthermore, CD200 mRNA expression strongly correlated in unsupervised hierarchical clustering with other melanoma signature transcripts, such as MITF, endothelin receptor type $B$, and silver bomolog. These results were independently confirmed in publicly available microarray data sets analyzing the NCI60 cell lines (38) and primary human melanocytic lesions (39) (Supplemental Figure $2, A$ and $B$ ). In the latter data set from Haqq and colleagues, relative CD200 mRNA expression was increased in melanocytic lesions and correlated with progression from nevi to melanoma (Supplemental Figure 2B), in accordance with our demonstration that CD200 mRNA is regulated by p-ERK. In the NCI60 data set, mRNA levels were uniformly higher in melanoma than any other tumor type, including the 8 representative leukemia cell lines (Supplemental Figure 2A). In aggregate, these data confirm that CD200 mRNA is highly and uniformly expressed in the majority of primary melanoma but only rarely in other solid tumors.

To confirm these results at the level of protein, we developed an immunohistochemical strategy to detect CD200 on FFPE samples. Upon validation of our immunohistochemical approach on known CD200-expressing tissues, placenta and thymus, we turned to the analysis of normal skin and 57 resected melanocytic lesions including benign and dysplastic nevi, primary melanomas, and melanoma metastases (Figure 2B). In accordance with the in vitro results (Figure 1A), no CD200 expression of quiescent melanocytes residing in normal skin was detected (Figure 2B). In contrast, however, there was marked expression in the majority of nevi and melanomas (Figure 2B and Table 1). Although CD200 is an ERK target in vitro and CD200 mRNA expression correlates with progression, CD200 expression as measured by immunohistochemistry (IHC) did not correlate with p-ERK staining in melanocytic lesions by IHC. In particular, CD200 expression was found throughout the entire spectrum of melanocytic neoplasms (Table 1), including nevi, whereas p-ERK expression was more frequent in later-stage lesions (data not shown). Several possible explanations could reconcile these findings: IHC analysis of p-ERK and CD200 is at best semiquantitative and not as reliable as RNA analysis; factors in addition to p-ERK likely regulate CD200 expression in nevi (see Discussion); or the increased proliferative rates of melanomas compared to nevi may dilute the protein expression of this stable cell-surface molecule (see below). These concerns notwithstanding, the IHC analyses demonstrate increased expression of CD200 protein in the majority of human melanocytic neoplasms.

$C D 200$ is required for inbibition of $T$ cell activation. In order to determine the functional importance of CD200 expression on melanoma cell lines, we sought to evaluate the generation of proinflam- 
Table 1

CD200 expression in melanocytic lesions by IHC

$\begin{array}{lc}\text { Lesion } & \text { CD200 staining (medium to high) } \\ \text { Nevi } & 27 \text { of } 34(79 \%) \\ \text { Melanoma } & 11 \text { of } 23(48 \%) \\ \text { Total } & 38 \text { of } 57(67 \%)\end{array}$

matory cytokines induced during a mixed lymphocyte response in the presence of melanoma cells with and without physiological levels of cell-surface CD200. Although CD200 mRNA rapidly plummeted with UO126 treatment (Figure 1B), no change was seen in the expression of CD200 on the cell surface even after 48 hours of UO126 treatment (data not shown), suggesting that the cell-surface fraction is highly stable. More prolonged treatment of cell lines with U0126 was not possible because of toxicity. In order to modulate CD200 expression in melanoma cells, we generated a short hairpin RNA (shRNA) construct specifically targeting the human CD200 ligand (Supplemental Figure 2A). shRNA oligonucleotides were cloned into a lentiviral transduction vector that also harbors EGFP driven by the CMV promoter. Therefore, melanoma cells transduced with the CD200 shRNA knockdown construct express GFP (Figure 3A). Maximal knockdown of human CD200 (>6.5 fold by flow cytometry; Supplemental Figure $3 \mathrm{~B}$ ) required 6 or more passages after transduction (more than 15 days after transduction; Figure 3B), suggesting that the membrane-associated fraction is stable and predominantly decreased on the cell surface by dilution with proliferation. CD200 knockdown did not alter the in vitro growth or survival of melanoma cell lines. After transduction and serial passage, transduced cells were flow sorted by GFP to produce cell lines that differed only in their expression of CD200 (Figure 3C and Supplemental Figure 3B). In order to control for potential effects of RNA interference, melanoma cell lines were also transduced with a nonspecific shRNA, which did not alter the levels of CD200 expression (Figure 3C).

To investigate the functional importance with regard to the immune response of CD200 expression on melanoma cell lines, cytokine production during an MLR was evaluated with the addition of irradiated melanoma cell lines with varying CD200 expression levels. When human melanoma cell lines WM2664, SKMEL24, and SKMEL28, which harbor high p-ERK and CD200 expression, were added in an MLR, an inhibitory effect on T cell activation by DCs was noted, as evidenced by a significant decrease in IL-2 and IFN- $\gamma$ levels (Figure 4A and Supplemental Figure 4A). In contrast, IL-2 and to a lesser extent IFN- $\gamma$ production was augmented when MLRs were performed in the presence of human melanoma cell lines PMWK, Mel505, and SKMEL187, which harbor low P-ERK and CD200 expression (Figure 1C and Supplemental Figure 4A).

As expression of many factors besides CD200 differed among these cell lines, we conducted MLRs with high-CD200-expressing melanoma cell lines WM2664, SKMEL24, and SKMEL28 in which the expression of CD200 was decreased by shRNA knockdown (WMKD, SK24KD, SK28KD, respectively). Inhibition of CD200 expression significantly impaired the ability of these cell lines to inhibit $\mathrm{T}$ cell proliferation when compared with the parental cell lines. In the presence of human melanoma cells transduced with CD200-knockdown shRNA, DC-activated lymphocytes produced increased levels of IL- 2 and IFN- $\gamma$ at 72 hours when compared with the parental cell lines or cells transduced with nonspecific shRNA (WMNS, SK24NS, SK28NS; Figure 4B and Supplemental Figure 4B). A comparable inhibition of $\mathrm{T}$ cell rosetting was associated with decreased CD200 expression across the panel of cell lines and with CD200 knockdown (Figure 4, C and D, and Supplemental Figure 5, A and B) in the MLRs. Therefore, physiological expression of CD200 on the cell surface of melanoma cell lines attenuates the ability of DCs to activate T cells.

\section{Discussion}

Using multiple melanoma cell lines, we have shown that expression of CD200 mRNA and protein is regulated by ERK activation, a signaling event required in the majority of melanomas for progression and maintenance. Through an analysis of CD200 mRNA expression in FFPE tumors, coupled with comparison of 2 independent publicly available melanoma microarray data sets, we have shown that expression of CD200 mRNA correlates with disease progression and appears more abundant in melanoma than any other common solid tumor or acute leukemia. Finally, we have shown that the endogenous CD200 expression on melanoma cell lines represses the ability of DCs to activate primary T cells in MLRs and that this effect is largely abolished by shRNA-mediated knockdown of CD200 in these lines. Therefore, the induction of CD200 by ERK activation appears to decrease tumor immunogenicity in melanoma.

Recent studies relying, like the present work, on unbiased, genome-wide analyses have suggested that CD200 expression may play a role in the pathogenesis of $\mathrm{B}$ cell lineage malignancies and acute leukemia. Moreaux et al. showed that increased CD200 mRNA expression by microarray correlated with reduced eventfree survival ( 22 months vs. 14 months) in patients with myeloma (40). The effect of CD200 on prognosis was independent of known adverse prognostic factors in myeloma, suggesting CD200 expression per se was pathogenic. A similar result was seen by Tonks et al. in acute myeloid leukemia (41). McWhirter et al. showed increased CD200 expression in chronic lymphocytic leukemia (CLL) samples relative to normal B cells. In accordance with our findings, the authors also showed that CD200 expression is associated with decreased CLL cell immunogenicity in an MLR (42). This effect could be partially blocked by incubation of the CLL cells with anti-CD200 antibodies, concordant with results seen using CD200 shRNA. In aggregate, these data suggest a pathogenic role of CD200 expression in certain hematologic malignancies.

We have extended these results to melanoma, an important human solid tumor for which ample evidence suggests there is a clinically beneficial antitumor immune response in some patients. In the NCI60 cell line data set (Supplemental Figure 2A) as well as FFPE samples (Figure 2A), CD200 mRNA expression was higher and more ubiquitous in melanoma compared with other solid tumors, although it is likely that other tumors in these data sets also harbor ERK activation. Therefore, although CD200 mRNA is induced by p-ERK in melanoma cell lines, this p-ERK responsiveness appears to be relatively restricted to melanocytic cell types. This finding would be consistent with the view that melanocyte-specific factors regulate CD200 mRNA expression in combination with p-ERK. It is worth noting, however, that increased CD200 expression was also detected in a small subset of samples of other solid tumors, such as breast, ovarian, and lung cancers (see Figure 2A and Supplemental Figure 2A). Therefore, CD200 expression may play a role in the disease pathogenesis of subsets of other solid tumors as well.

We believe these findings have important and direct clinical relevance to the treatment of melanoma. For example, while there is 
A

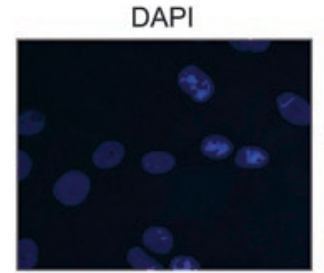

B
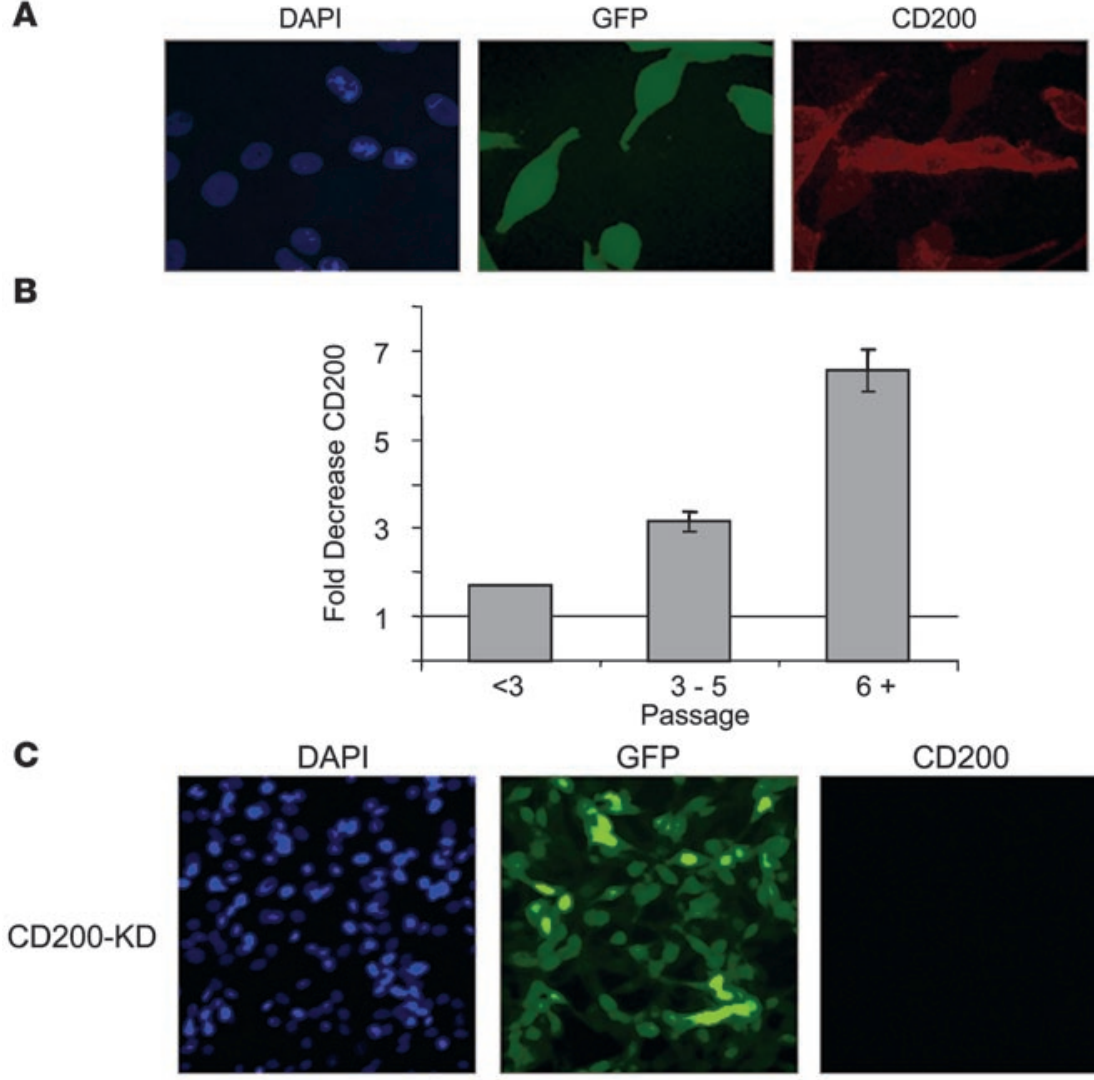

GFP
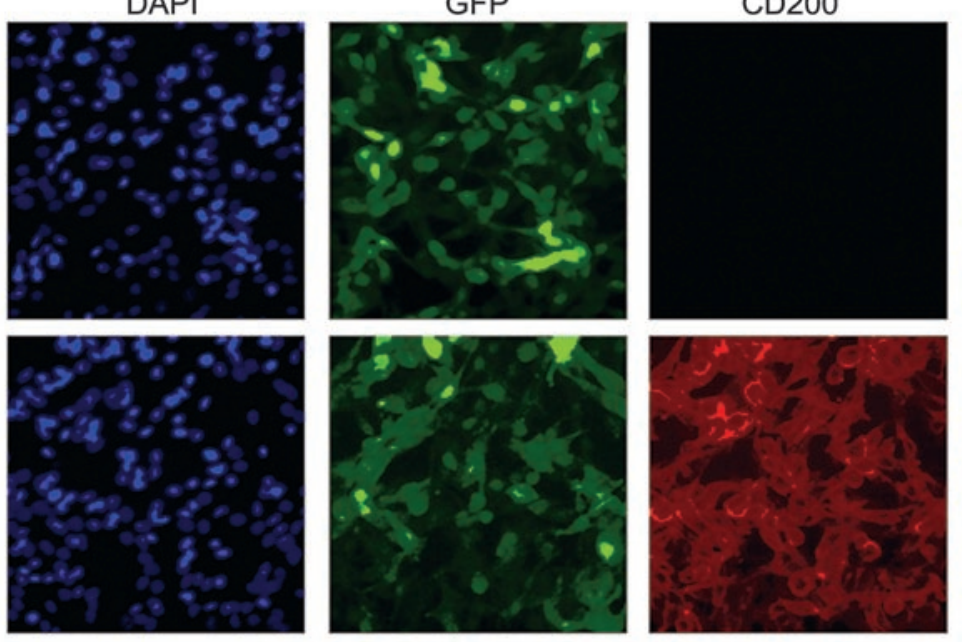
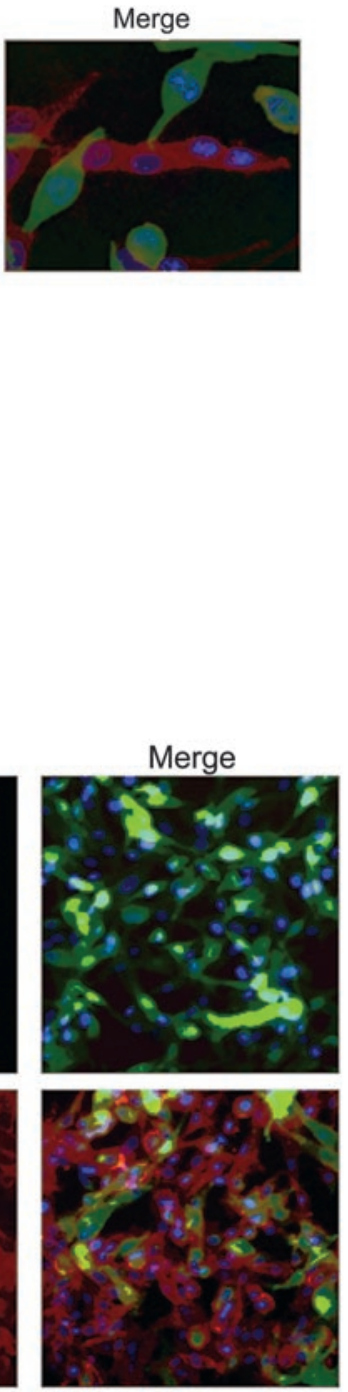

Figure 3

shRNA-mediated knockdown of CD200 expression. (A) WM2664 human melanoma cells were transduced with a GFP-expressing human CD200 shRNA construct and stained with an anti-human CD200 antibody (red). Untransduced WM2664 cells expressed CD200 (red). Transduced WM2664 cells were negative for CD200 expression. Nuclei of cells are stained with DAPI (blue). Original magnification, $\times 40$. (B) CD200 expression at different passages in culture after transduction was analyzed by flow cytometry. Efficient knockdown of the CD200 protein was observed after 6 passages ( $>15$ days) in culture. (C) WM2664 human melanoma cells were FACS sorted for GFP expression and then analyzed by immunofluorescence for CD200 expression. WM2664 cells transduced with a CD200 shRNA were negative for CD200 expression. WM2664 cells transduced with a nonspecific shRNA showed intense staining for CD200 (red). Original magnification, $\times 10$.

some enthusiasm in the field of melanoma therapy to combine novel RAF and MEK inhibitors, which inhibit proliferation, with classical chemotherapeutic agents, which target cycling cells, our data suggest that the combination of such targeted kinase inhibitors with immunomodulatory agents such as IL-2 or IFN is at least as rational a strategy. Moreover, as at least one CD200 blocking antibody is in preclinical development for cancer therapy (Alexion Pharmaceuticals), this work has important implications for the development of anticancer therapies to target directly the CD200-CD200R interaction. While blocking antibodies against CTLA4 have shown promising single-agent activity in melanoma, it is important to note that response to anti-CTLA4 seems to correlate with the development of significant immune enterocolitis (15). Patients who had the most severe autoimmune stigmata appeared to derive the greatest benefit from CTLA4 blockade. In this regard, it is encouraging to note that the CD200-knockout mice demonstrate a much less severe autoimmune phenotype than CTLA4-deficient mice $(25,43$, 44), suggesting that blockade of CD200 will be better tolerated than that of CTLA4. Last, we believe these data suggest that melanoma is perhaps the best candidate disease for the testing and development of anti-CD200 approaches. In particular, patients with melanoma who are candidates for phase I therapies are not rare and tend to be younger, less heavily pretreated, and of better performance status than patients with refractory hematologic malignancies, who are uncommon and generally heavily pretreated prior to consideration for a phase I trial. Therefore, we believe this identification of mela- 
A

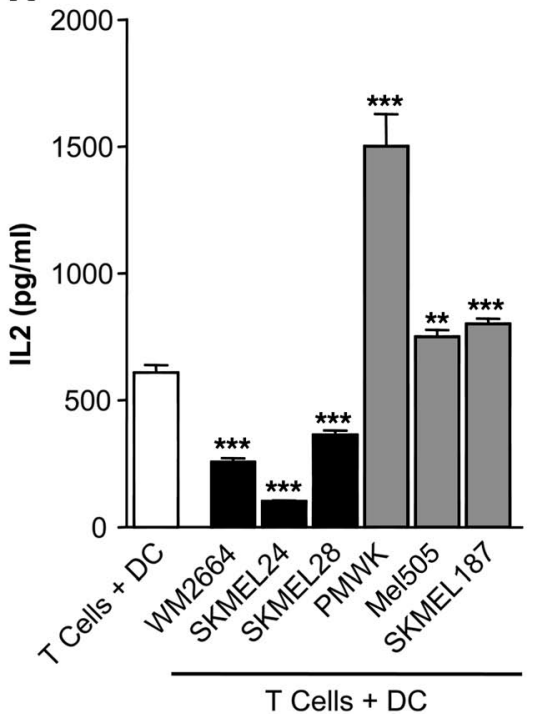

B

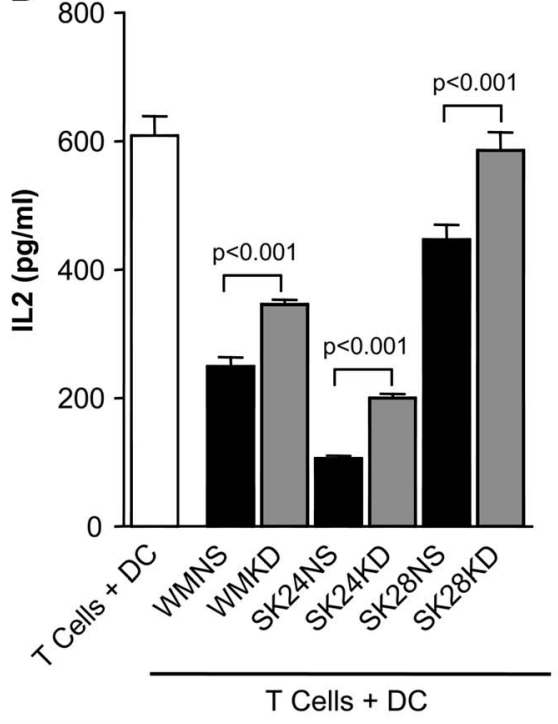

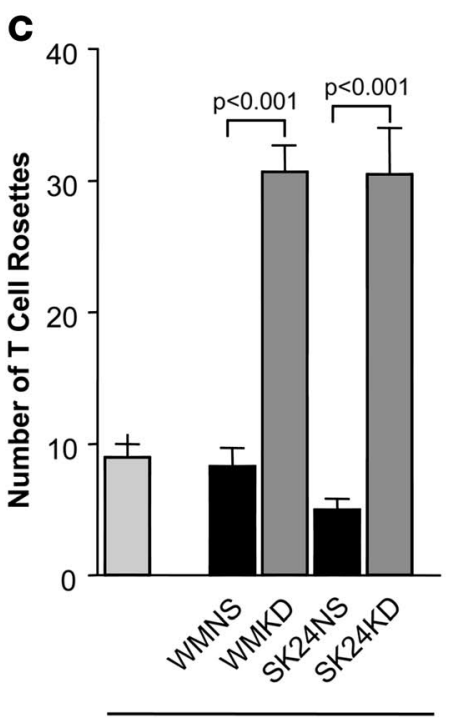

T Cells + DCs

D

WM2664

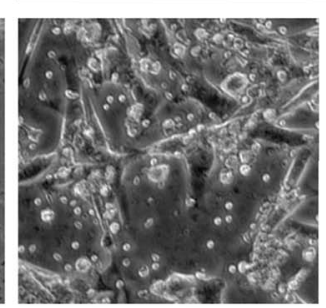

Non-specific

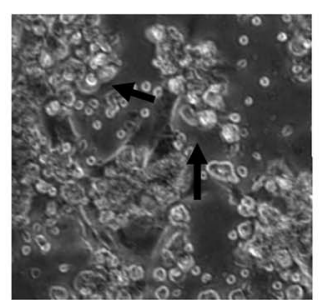

CD200 KD

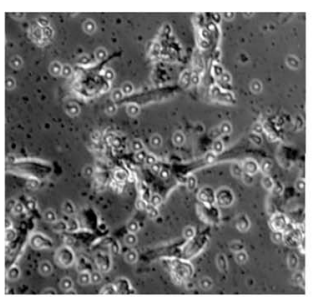

Non-specific

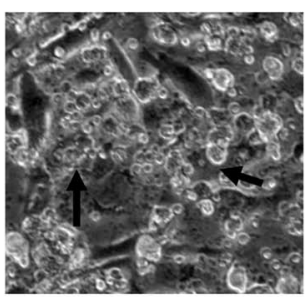

CD200 KD

T Cells + DCs

\section{Figure 4}

CD200 is required for T cell repression. (A) IL-2 production by T lymphocytes during MLRs with the addition of human melanoma cell lines with varying levels of CD200 expression. WM2664, SKMEL24, and SKMEL28 express high levels of CD200; PMWK, Mel505, and SKMEL187 express low levels of CD200. IL-2 production, as a marker of T cell activation, was determined by ELISA after 72 hours of incubation. Indicated statistical comparisons are between the indicated cell line versus T cells plus DCs only ( $\left.{ }^{\star \star} P<0.005 ;{ }^{* \star *} P<0.0001\right)$. Error bars are \pm SEM. (B) MLRs in the presence of WM2664, SKMEL24, and SKMEL28 transduced with nonspecific hairpin (WMNS, SK24NS, SK28NS) and WM2664, SKMEL24, and SKMEL28 transduced with CD200 knockdown (WMKD, SK24KD, SK28KD). DCs and T cells were mixed with the indicated human melanoma cell lines with or without CD200 shRNA knockdown. IL-2 production was significantly higher in CD200-knockdown melanoma cell lines (WMKD, SK24KD, SK28KD) when compared with the parental melanoma cell lines transduced with a nonspecific shRNA (WMNS, SK24NS, and SK28NS). Error bars are \pm SEM. (C) Quantification of T cell rosettes. WMKD and SK24KD show a significant increase in the formation of T cell rosettes when compared with their parental cell lines transduced with a nonspecific shRNA in MLRs. A mix of T cells and DCs was used as a positive control (far-left bar). Error bars are \pm SEM. (D) WMKD and SK24KD show a significant increase in the formation of T cell rosettes when compared with their parental cell lines transduced with a nonspecific shRNA in MLRs. A mix of T cells and DCs was used as a positive control (far-left panel). Original magnification, $\times 100$.

noma as a rational target disease will facilitate the development of CD200-CD200R anticancer therapies.

\section{Methods}

Cell lines. Human melanoma cell lines WM2664 and PMWK were obtained from J. Arbiser (Emory University, Atlanta, Georgia, USA); Mel505 was obtained from J. Hansson (Karolinska Institute, Stockholm, Sweden); SKMEL187 was obtained from A. Houghton (Memorial Sloan-Kettering Cancer Center, New York, New York, USA); and SKMEL24, SKMEL28, and 293FT were obtained from ATCC. Cells were cultured in either DMEM with the addition of 10\% FBS and penicillin/streptomycin (WM2664, 293FT, PMWK, SKMEL187), MEM- $\alpha$ with the addition of $10 \%$ nonessential amino acids and 10\% FBS and penicillin/streptomycin (SKMEL24, SKMEL28), or RPMI with addition of $10 \%$ FBS and penicillin/streptomy- cin (Mel505) (Gibco; Invitrogen). Human melanoma cell lines, WM2664, SKMEL24, SKMEL28, SKMEL187, and Mel505, were derived from vertical growth phase melanomas, and human melanoma cell line PMWK was derived from a radial growth phase melanoma. Cells were incubated at $37^{\circ} \mathrm{C}, 5 \% \mathrm{CO}_{2}$, and medium was changed every other day. Subconfluent cells were trypsinized and replated at the appropriate densities. Human DCs were differentiated using $\mathrm{CD} 34^{+}$selection (Miltenyi Biotec) following a 15-1 apheresis collection of peripheral blood (Cobe-Spectra; GambroBCT). DCs were expanded first in the presence of GM-CSF (Immunex), FLT3-ligand, IL-4, stem cell factor, and TNF- $\alpha$ (Peprotech) using AIM-V media (Gibco; Invitrogen) with $10 \%$ human $\mathrm{AB}$ serum (Gemini Bio-Products). Microarray analysis, TaqMan (Applied Biosciences) analysis, ERK and BRAF Western blot analysis, and ERK inhibition with UO126 of melanoma cell lines were performed as described previously $(2,45)$. 
MLRs. MLRs were set up in 12 -well plates using $1 \times 10^{5} \mathrm{DCs}$ and $2 \times 10^{6} \mathrm{~T}$ cells. T cells were enriched by incubating the cells for 2 hours in tissue culture flasks and taking the nonadherent cell fraction. Irradiated melanoma cell lines (250,000 per reaction) were added to the MLRs at the start of the experiment, and supernatants were collected after 72 hours and analyzed for the presence of IL-2 and IFN- $\gamma$.

$I L-2$ and IFN- $\gamma$ ELISA. Conditioned medium was obtained from mixed lymphocytic reactions and assayed for the presence of IL- 2 and IFN- $\gamma$ by ELISA following the manufacturer's instructions (Quantikine Kit; R\&D Systems). OD measurements were performed using a Kinetic Microplate Reader (Molecular Devices). T cells plus DCs in the absence of melanoma cells and melanoma cells alone (data not shown) were used as controls.

DNA constructs, viral production, and infection of target cells. shRNA coding oligonucleotides were designed and verified to be specific for human CD200 according to a BLAST search (http://www.ncbi.nlm.nih.gov/blast/) against human genome. The human CD200 shRNA targeting sequence was 5'-TGGTAATTCTTCTCGTCCTGTTCAAGAGATAGGACGAGAAGAATTACCTTTTTTTTC- $3^{\prime}$. The insert was cloned between the XhoI and HpaI sites of the pLentiLox 3.7 vector. A nonspecific shRNA construct was used as control. The production of lentiviruses in 293FT cells and infection of target cells were as described previously (46). WM2664 and SKMEL24 cells were infected with the viral supernatant, CD200 expression levels were analyzed, and positive cells were sorted by flow cytometry. BRAF mutant shRNA (pSuper) plasmid or scrambled nonspecific shRNA (pSuper) plasmid, as previously described (45), was added to 293FT cells for the production of retrovirus. WM2664, SKMEL24, and SKMEL28 cells were infected with viral supernatant for 24 hours; cells were selected in media containing $5 \mu \mathrm{g} / \mathrm{ml}$ puromycin for 4-5 days; and CD200 expression levels were analyzed by TaqMan quantitative RT-PCR.

Flow cytometry. Exponentially growing cells were detached using $0.5 \mathrm{mM}$ EDTA for 3-5 minutes at $37^{\circ} \mathrm{C}$ and stained with FITC-conjugated antibodies against the CD200 surface antigen (MCA1958FA; Serotec) analyzed using a FACScan analytical flow cytometer (BD Biosciences - Immunocytometry Systems). Appropriately gated cells were analyzed (10,000 cells counted per staining) and compared with unstained or isotype controls.

Indirect immunofluorescence analysis and image acquisition. Cells were plated on glass coverslips and fixed with 4\% PFA. After washing and blocking with PBS plus $10 \%$ FBS, cells were stained with mouse anti-human CD200 antibody (1:100 dilution; R\&D Systems), followed by incubation with ${ }^{594} \mathrm{AlexaFluor}-\mathrm{conjugated}$ goat anti-mouse secondary antibody (Neomarkers). Cell-containing coverslips were mounted onto glass slides, and nuclei were counterstained using DAPI-Vectashield (Vector Laboratories). Cells were visualized and photographed using a fluorescence microscope (model IX-81; Olympus) equipped with an X60 1.45 NA objective, a CCD camera (model C4742-80-12AG; Hamamatsu), and controlled by AQM Advanced 6 software (Kinetic Imaging Ltd.).

RNA analysis and IHC on nevi and melanomas. RNA from FFPE tumors samples of 2-8 years of age was prepared using a commercially available
FFPE extraction kit (Arcturus) and amplified and labeled as described previously (37). Amplified RNA from tumors was hybridized to the Agilent $22 \mathrm{~K} 3$ '-biased arrays and analyzed by unsupervised hierarchical clustering. An excerpted cluster of this analysis is shown in Figure 2A. See ref. 37 for details and primary data; all primary microarray data can be downloaded from the UNC microarray database (see the "published data" link; https:// genome.unc.edu/).

The study was approved by the Institutional Review Board of the University of North Carolina. The tissues used in this study were selected at random from a series of cases treated at UNC Hospitals after 2000. All cases, including 23 melanomas and 34 benign nevi, underwent standardized histopathology review of H\&E-stained sections of the specimens for confirmation of diagnosis and evaluation of lesional characteristics. Five-micrometer sections were deparaffinized and stained using a commercially available CD200 antibody (AF2724; R\&D) at a dilution of 1:50 with immunodetection by HRP after Tris-EDTA antigen retrieval. Staining was validated on control tissues (placenta and thymus) consistent with the known pattern of expression of CD200 in these organs. All tissues were scored for staining intensity for localization, percentage of cells, and intensity of staining. For the majority of nevi and melanomas, more than $50 \%$ of the cells stained with the CD200 antibody. The CD200 localization was cytoplasmic or both cytoplasmic and membranous. The intensity was scored as none, low, medium, or high and dichotomized as none-to-low versus medium-to-high for the purposes of this article.

Statistics. For comparisons shown in Figures 1 and 4, a 2-tailed Student's $t$ test was used. A $P$ value of less than 0.05 was considered significant. Analyses were performed using GraphPad Prism Software version 3.0.

\section{Acknowledgments}

The authors wish to acknowledge Robert Sandler, Tope Keku, and Charles Perou for advice and assistance; and Courtney Boyd and the AP Translational Core Laboratory, under the direction of Leigh Thorne, for assistance with the CD200 immunohistochemical analysis. This work was supported by grants from the NCI and NIEHS (CA106991, CA90679, CA105837, and ES14635), the CALGB (to S. Penland), The Golfers Against Cancer Foundation (to N.E. Sharpless and J.E. Bear), the National Cancer Center (to K.E. Petermann), and the Sidney Kimmel Cancer Foundation (to N.E. Sharpless).

Received for publication March 19, 2007, and accepted in revised form September 19, 2007.

Address correspondence to: Norman E. Sharpless, The Lineberger Comprehensive Cancer Center, CB \#7295, Departments of Medicine and Genetics, University of North Carolina School of Medicine, Chapel Hill, North Carolina 27599-7295, USA. Phone: (919) 966-1185 or (919) 966-4067; Fax: (919) 966-8212; E-mail: nes@ med.unc.edu.
1. Chin, L., Garraway, L.A., and Fisher, D.E. 2006. Malignant melanoma: genetics and therapeutics in the genomic era. Genes Dev. 20:2149-2182.

2. Shields, J.M., et al. 2007. Lack of extracellular signal-regulated kinase mitogen-activated protein kinase signaling shows a new type of melanoma. Cancer Res. 67:1502-1512.

3. Chin, L., et al. 1999. Essential role for oncogenic Ras in tumour maintenance. Nature. 400:468-472.

4. Solit, D.B., et al. 2006. BRAF mutation predicts sensitivity to MEK inhibition. Nature. 439:358-362.

5. Slingluff, C.L., Jr., et al. 2006. Immunity to melanoma antigens: from self-tolerance to immunotherapy. Adv. Immunol. 90:243-295.
6. Kirkwood, J.M., Moschos, S., and Wang, W. 2006. Strategies for the development of more effective adjuvant therapy of melanoma: current and future explorations of antibodies, cytokines, vaccines, and combinations. Clin. Cancer Res. 12:2331s-2336s.

7. Romero, P., Cerottini, J.C., and Speiser, D.E. 2006 The human $\mathrm{T}$ cell response to melanoma antigens. Adv. Immunol. 92:187-224.

8. Ribas, A. 2006. Update on immunotherapy for melanoma. J. Natl. Compr. Canc. Netw. 4:687-694.

9. Rosenberg, S.A. 2001. Progress in human tumour immunology and immunotherapy. Nature. 411:380-384

10. Boasberg, P.D., et al. 2006. Enhanced survival asso- ciated with vitiligo expression during maintenance biotherapy for metastatic melanoma. J. Invest. Dermatol. 126:2658-2663.

11. Nordlund, J.J., et al. 1983. Vitiligo in patients with metastatic melanoma: a good prognostic sign. J. Am. Acad. Dermatol. 9:689-696.

12. Oyarbide-Valencia, K., et al. 2006. Therapeutic implications of autoimmune vitiligo T cells. Autoimmun. Rev. 5:486-492.

13. O’Day, S., and Boasberg, P. 2006. Management of metastatic melanoma 2005. Surg. Oncol. Clin. N. Am. 15:419-437.

14. Hodi, F.S., et al. 2003. Biologic activity of cytotoxic T lymphocyte-associated antigen 4 antibody block- 
ade in previously vaccinated metastatic melanoma and ovarian carcinoma patients. Proc. Natl. Acad. Sci. U. S. A. 100:4712-4717.

15. Beck, K.E., et al. 2006. Enterocolitis in patients with cancer after antibody blockade of cytotoxic T-lymphocyte-associated antigen 4. J. Clin. Oncol. 24:2283-2289.

16. Bennicelli,J.L., and Guerry, D.T. 1993. Production of multiple cytokines by cultured human melanomas. Exp. Dermatol. 2:186-190.

17. Chen, Q., Daniel, V., Maher, D.W., and Hersey, P. 1994. Production of IL-10 by melanoma cells: examination of its role in immunosuppression mediated by melanoma. Int. J. Cancer. 56:755-760.

18. Curiel-Lewandrowski, C., and Demierre, M.F. 2000. Advances in specific immunotherapy of malignant melanoma. J. Am. Acad. Dermatol. 43:167-185.

19. Capobianco, A., Rovere-Querini, P., Rugarli, C., and Manfredi, A.A. 2006. Melanoma cells interfere with the interaction of dendritic cells with NK/LAK cells. Int. J. Cancer. 119:2861-2869.

20. Hersey, P., et al. 2004. Phase I/II study of treatment with dendritic cell vaccines in patients with disseminated melanoma. Cancer Immunol. Immunother. 53:125-134.

21. Palucka, A.K., et al. 2006. Dendritic cells loaded with killed allogeneic melanoma cells can induce objective clinical responses and MART-1 specific CD8+ T-cell immunity. J. Immunother. 29:545-557.

22. Barclay, A.N., Clark, M.J., and McCaughan, G.W. 1986. Neuronal/lymphoid membrane glycoprotein MRC OX-2 is a member of the immunoglobulin superfamily with a light-chain-like structure. Biochem. Soc. Symp. 51:149-157.

23. Wright, G.J., et al. 2003. Characterization of the CD200 receptor family in mice and humans and their interactions with CD200. J. Immunol. 171:3034-3046.

24. Gorczynski, R., et al. 2004. CD200 is a ligand for all members of the CD200R family of immunoregulatory molecules. J. Immunol. 172:7744-7749.
25. Hoek, R.M., et al. 2000. Down-regulation of the macrophage lineage through interaction with OX2 (CD200). Science. 290:1768-1771.

26. Cherwinski, H.M., et al. 2005. The $\mathrm{CD} 200$ receptor is a novel and potent regulator of murine and human mast cell function. J. Immunol. 174:1348-1356.

27. Shiratori, I., et al. 2005. Down-regulation of basophil function by human CD200 and human herpesvirus-8 CD200. J. Immunol. 175:4441-4449.

28. Jenmalm, M.C., Cherwinski, H., Bowman, E.P., Phillips, J.H., and Sedgwick, J.D. 2006. Regulation of myeloid cell function through the CD200 receptor. J. Immunol. 176:191-199.

29. Zhang, S., Cherwinski, H., Sedgwick, J.D., and Phillips, J.H. 2004. Molecular mechanisms of CD200 inhibition of mast cell activation. J. Immunol. 173:6786-6793.

30. Voigt, S., Sandford, G.R., Hayward, G.S., and Burns, W.H. 2005. The English strain of rat cytomegalovirus (CMV) contains a novel captured CD200 (vOX2) gene and a spliced CC chemokine upstream from the major immediate-early region: further evidence for a separate evolutionary lineage from that of rat CMV Maastricht. J. Gen. Virol. 86:263-274.

31. Foster-Cuevas, M., Wright, G.J., Puklavec, M.J., Brown, M.H., and Barclay, A.N. 2004. Human herpesvirus $8 \mathrm{~K} 14$ protein mimics CD200 in downregulating macrophage activation through CD200 receptor. J. Virol. 78:7667-7676.

32. Zhang, J., Wang, J., Wood, C., Xu, D., and Zhang, L. 2005. Kaposi's sarcoma-associated herpesvirus/ human herpesvirus 8 replication and transcription activator regulates viral and cellular genes via interferon-stimulated response elements. J. Virol. 79:5640-5652.

33. Cameron, C.M., Barrett, J.W., Liu, L., Lucas, A.R., and McFadden, G. 2005. Myxoma virus M141R expresses a viral CD200 (vOX-2) that is responsible for down-regulation of macrophage and T-cell activation in vivo. J. Virol. 79:6052-6067.

34. Chang,Y.,etal. 1996. Cyclinencoded by KS herpesvirus.
Nature. 382:410.

35. Fragnet, L., Blasco, M.A., Klapper, W., and Rasschaert, D. 2003. The RNA subunit of telomerase is encoded by Marek's disease virus. J. Virol. 77:5985-5996.

36. Dyson, N., Howley, P.M., Munger, K., and Harlow, E. 1989. The human papilloma virus-16 E7 oncoprotein is able to bind to the retinoblastoma gene product. Science. 243:934-937.

37. Penland, S.K., et al. 2007. RNA expression analysis of formalin-fixed paraffin-embedded tumors. Lab. Invest. 87:383-391.

38. Ross, D.T., et al. 2000. Systematic variation in gene expression patterns in human cancer cell lines. Nat. Genet. 24:227-235.

39. Haqq, C., et al. 2005. The gene expression signatures of melanoma progression. Proc. Natl. Acad. Sci. U. S. A. 102:6092-6097.

40. Moreaux, J., et al. 2006. CD200 is a new prognostic factor in multiple myeloma. Blood. 108:4194-4197.

41. Tonks, A., et al. 2007. CD200 as a prognostic factor in acute myeloid leukaemia. Lenkemia. 21:586-568.

42. McWhirter, J.R., et al. 2006. Antibodies selected from combinatorial libraries block a tumor antigen that plays a key role in immunomodulation. Proc. Natl. Acad. Sci. U. S. A. 103:1041-1046.

43. Tivol, E.A., et al. 1995. Loss of CTLA-4 leads to massive lymphoproliferation and fatal multiorgan tissue destruction, revealing a critical negative regulatory role of CTLA-4. Immunity. 3:541-547.

44. Waterhouse, P., et al. 1995. Lymphoproliferative disorders with early lethality in mice deficient in Ctla-4. Science. 270:985-988.

45. Baines, A.T., et al. 2005. Use of retrovirus expression of interfering RNA to determine the contribution of activated $\mathrm{k}$-ras and ras effector expression to human tumor cell growth. Methods Enzymol. 407:556-574.

46. Rubinson, D.A., et al. 2003. A lentivirus-based system to functionally silence genes in primary mammalian cells, stem cells and transgenic mice by RNA interference. Nat. Genet. 33:401-406. 